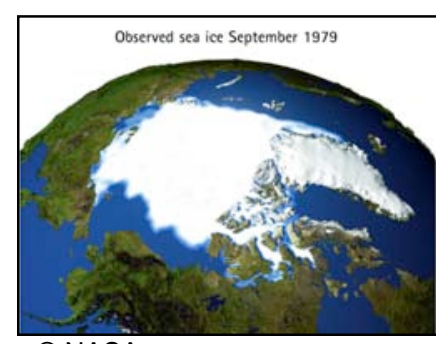

(C) NASA

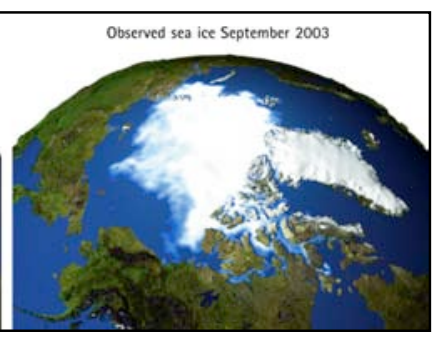

(C) Tony Weyiouanna, Sr.

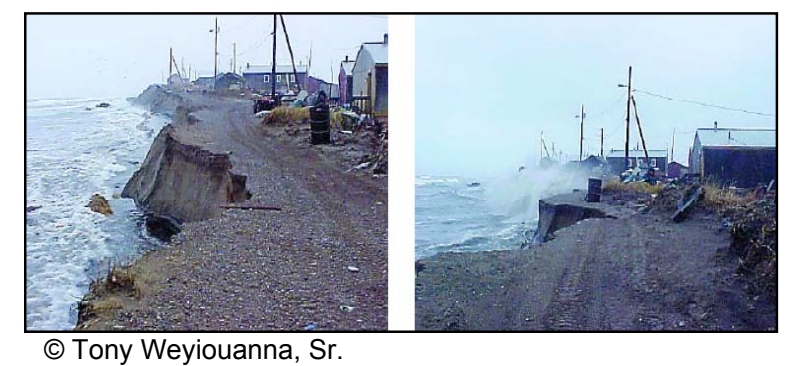

\title{
ALASKAN OPINIONS ON GLOBAL WARMING
}

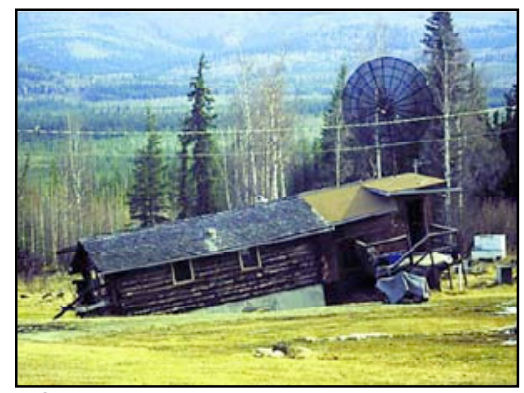

(c) Larry Hinzman

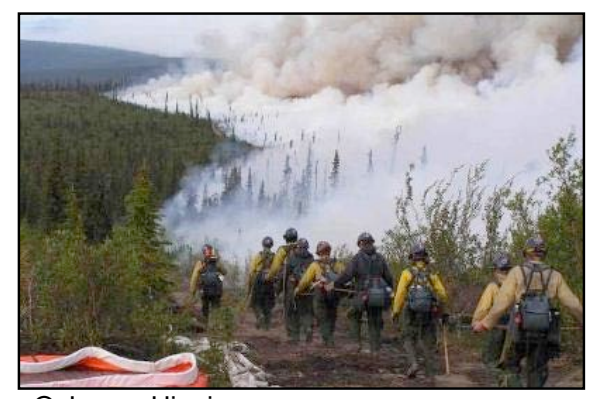

(C) James Higgins

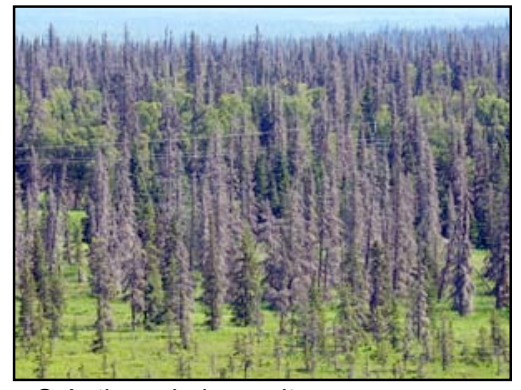

(C) Anthony Leiserowitz

Principal Investigators ${ }^{1}$ :

Dr. Anthony Leiserowitz

Decision Research \&

The Center for Research on Environmental Decisions (CRED) at Columbia University

Jean Craciun

Craciun Research Group, Inc.

\footnotetext{
${ }^{1}$ Cite as: Leiserowitz, A., \& Craciun, J. (2006). Alaskan Opinions on Global Warming (No. 06-10). Eugene: Decision Research. http://www.decisionresearch.org/Projects/Climate_Change/
} 


\section{Alaskan Opinions on Global Warming}

\section{Table of Contents}

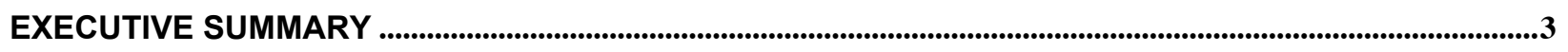

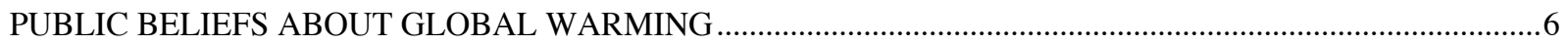

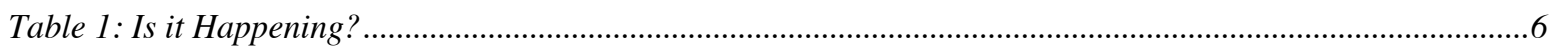

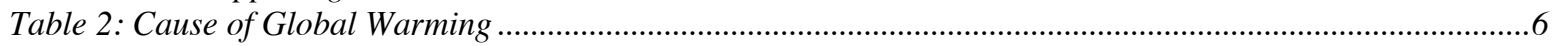

Table 3: Current Impacts ..............................................................................................................

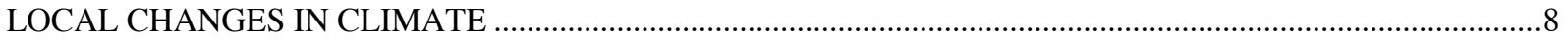

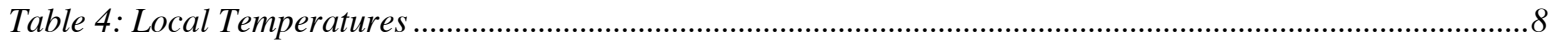

Table 5: Cause of Local Temperature Changes ………............................................................................

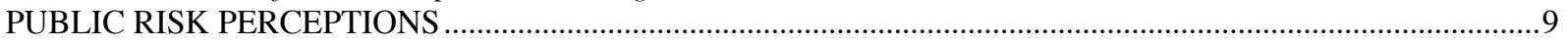

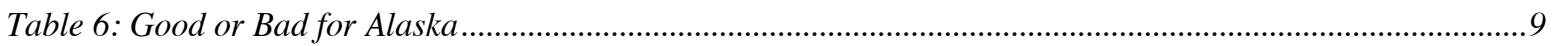

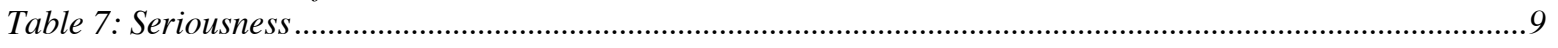

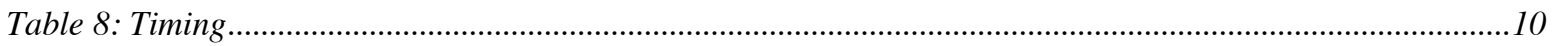

Table 9: Likelihood of Impacts on Alaska ..............................................................................................10

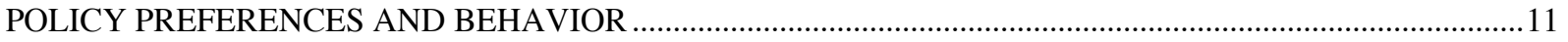

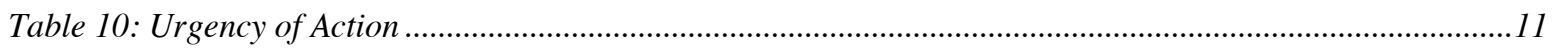

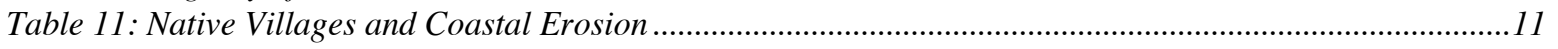

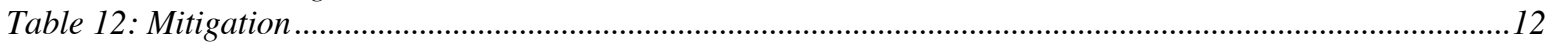

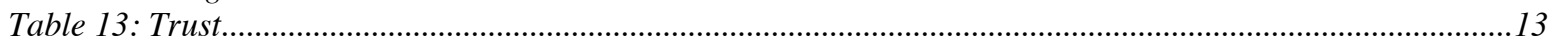

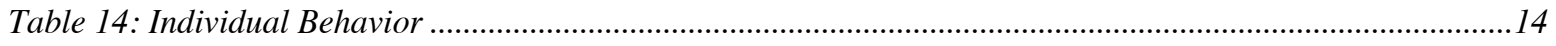

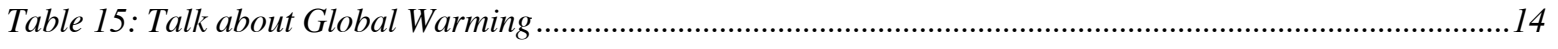

SELECTED RESULTS BY POLITICAL PARTY, POLITICAL IDEOLOGY, AND REGION ..............................15

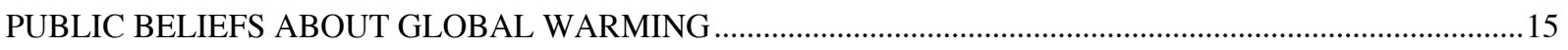

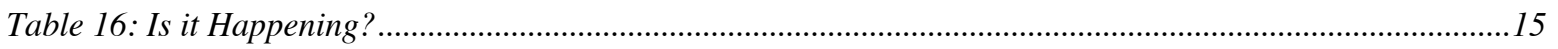

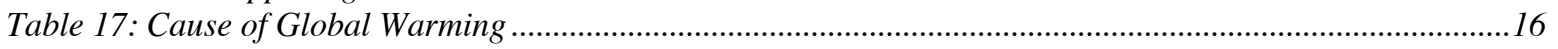

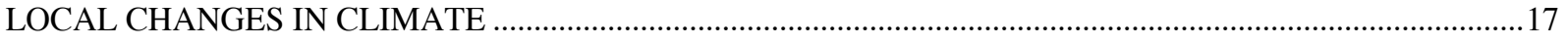

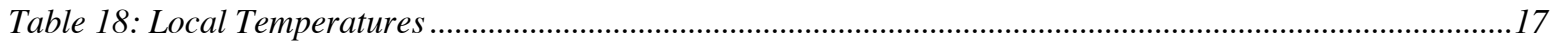

Table 19: Cause of Local Temperature Changes …………….......................................................................18

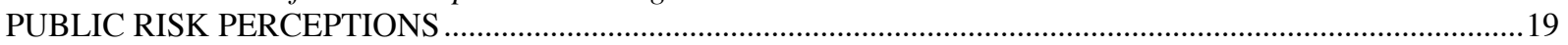

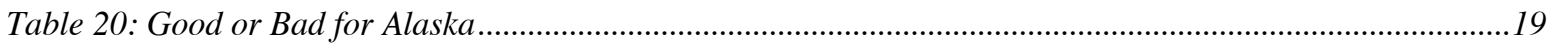

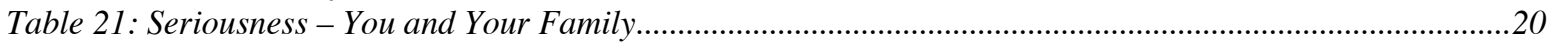

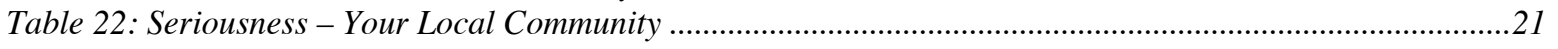

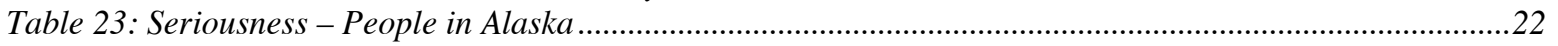

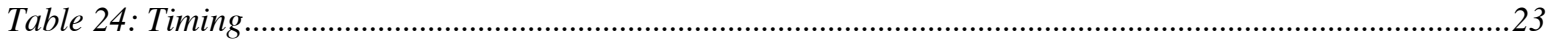

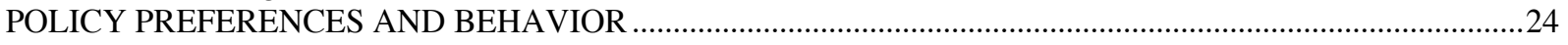

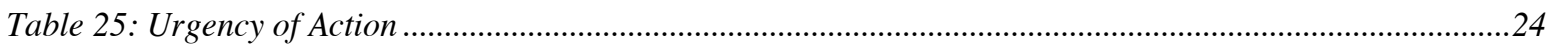

Table 26: Native Villages and Coastal Erosion ..............................................................................................

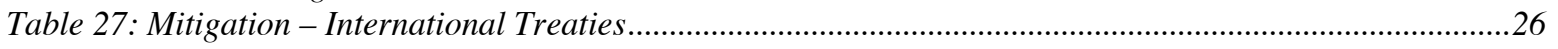

Table 28: Mitigation - Regulation of Power Plants ……….........................................................................27

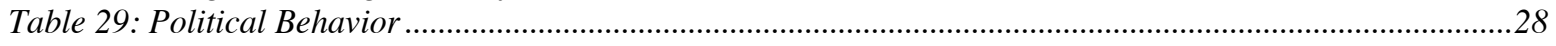

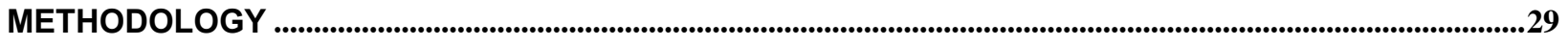

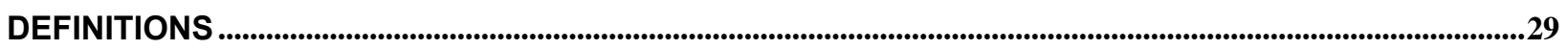




\section{Executive Summary}

A statewide, representative telephone survey entitled "Alaskan Opinions on Global Warming" was conducted with 1,016 Alaskan adults May 9 - June 29, 2006.

\section{The results show that most Alaskans consider global warming a serious threat.}

The study, funded by the National Science Foundation, was conducted in Alaska by Craciun Research Group ${ }^{2}$ as part of a scientific study commissioned by Dr. Anthony Leiserowitz of Decision Research ${ }^{3}$ through the Center for Research on Environmental Decisions (CRED) ${ }^{4}$. Highlights of the survey:

- Over $81 \%$ of Alaskans are convinced that global warming is happening.

- A majority (55\%) believe it is caused primarily by human activities such as the burning of fossil fuels, as opposed to normal cycles in the earth's environment (37\%).

- Most Alaskans believe global warming is already causing or accelerating the loss of sea ice $(83 \%)$ melting permafrost $(82 \%)$, coastal erosion $(74 \%)$, and forest fires $(72 \%)$ in Alaska, among other impacts.

- A large majority (67\%) report that their local temperatures have increased, while $93 \%$ of people who have noticed local temperature changes say that global warming is at least partly responsible.

- Two out of three Alaskans (67\%) say that global warming will be bad for Alaska, while $26 \%$ say it will be good.

- Majorities of Alaskans believe that global warming is a serious threat to themselves and their family (55\%), their local community (59\%), other countries $(68 \%)$, Alaska as a whole $(71 \%)$, the United States $(71 \%)$, and plants and animals $(76 \%)$.

- Many (43\%) expect that global warming will have dangerous impacts on Alaskans within the next 10 years.

- Majorities expect that over the next 50 years global warming will cause increased flooding of towns and villages (73\%), worse storms (68\%), fewer salmon $(67 \%)$, and extinction of the polar bear (65\%). Majorities also believe, however, that global warming

\footnotetext{
${ }^{2}$ Craciun Research Group, Inc. is a full service market research company committed to excellence in research design and analysis for twenty-six years with offices in Anchorage, Alaska; Washington DC; and Seattle, Washington (www.crgresearch.com).

${ }^{3}$ Decision Research is a non-profit, scientific research institute dedicated to helping individuals, industry, government, and society understand and cope with the complex and often risky decisions of modern life (www.decisionresearch.org).

${ }^{4}$ The Center for Research on Environmental Decisions (CRED) is an interdisciplinary center that studies individual and group decision making under climate uncertainty and decision making in the face of environmental risk. CRED's objectives address the human responses to climate change and climate variability as well as improved communication and increased use of scientific information on climate variability and change. Major funding is provided under the cooperative agreement NSF SES-0345840 (www.cred.columbia.edu).
} 
is likely to provide more comfortable temperatures $(73 \%)$, and more tourism in Alaska $(60 \%)$.

- Nearly all Alaskans (93\%) believe global warming is a problem. However, roughly half $(48 \%)$ believe it is an urgent problem requiring immediate government action while half $(45 \%)$ believe it is a longer-term problem requiring more study first.

- A plurality of Alaskans ( $41 \%)$ believe the state of Alaska should help native villages facing severe coastal erosion move to safer ground.

- Most Alaskans support the regulation of greenhouse gas emissions from power plants $(81 \%)$ and the signing of international treaties to reduce emissions $(71 \%)$, but oppose higher taxes on electricity $(71 \%)$ or gasoline $(69 \%)$.

- Alaskans primarily trust their friends and family (86\%), scientists ( $82 \%)$, and environmental groups $(63 \%)$ to tell them the truth about global warming, but distrust President Bush (66\%), Alaskan politicians (70\%), and corporations (75\%).

- Most Alaskans say that, due to their concern about global warming, they are likely to buy energy-efficient appliances $(73 \%)$ or contact their politicians $(60 \%)$, while nearly half (48\%) say they are likely to join, donate money to, or volunteer with an organization working on issues related to global warming.

- Two-thirds $(62 \%)$ report that they very often or occasionally talk about global warming with their friends and family.

- Democrats and liberals express stronger concern about global warming than do Republicans and conservatives.

- Alaskans without a party preference and moderates show levels of concern more similar to Democrats and liberals than to Republicans and conservatives.

- Majorities of Republicans and conservatives are convinced that global warming is happening ( $65 \%$ and $68 \%$ respectively), will be bad for Alaska (58\% and $59 \%)$, is a serious threat to people in Alaska (62\% and $60 \%)$, support international treaties to reduce greenhouse gas emissions $(61 \%$ and $53 \%)$, and support the regulation of greenhouse gas emissions from power plants $(82 \%$ and $76 \%)$.

- Majorities of Republicans and conservatives, however, believe that global warming is caused primarily by natural cycles, is not a serious threat to themselves, their families, or their local communities, and is a longer-term problem requiring more study before government action.

- More Semi-Urban and Rural Alaskans believe that global warming is currently dangerous and a serious threat to themselves, their families, and local communities than do Urban or Northern Urban Alaskans. ${ }^{5}$

\footnotetext{
${ }^{5}$ See the "Definitions" section at the end of the report for descriptions of these categories.
} 


\section{Principal Investigators:}

Dr. Anthony Leiserowitz is a research scientist at Decision Research and a principal investigator at the Center for Research on Environmental Decisions (CRED) at Columbia University.

Dr. Anthony Leiserowitz

Decision Research

1201 Oak Street

Eugene, OR 97401

tel.: (541) 485-2400

fax: (541) 485-2403

ecotone@uoregon.edu

Jean Craciun is the CEO/President of Craciun Research Group, Inc. (dba nationally as CRG Research); she is a Sociologist and an internationally recognized trainer in the market research industry.

Jean Craciun

Craciun Research Group, Inc.

1334 K. Street, Suite B

Anchorage, Alaska 99501

direct 907.279.3901

main 907.279 .3982

fax 907.279.0321

jcraciun@crgresearch.com

Seattle Washington office

600 Stewart Street, Suite 1300

Seattle Washington

tel.: 206-443-8346

fax: $206-382-1338$

Washington DC office

1730 Rhode Island Avenue NW, Suite 715

Washington DC 20036

tel.: 202-862-5566

fax: 202-232-0930 


\section{Results}

\section{PUBLIC BELIEFS ABOUT GLOBAL WARMING}

\section{Table 1: Is it Happening?}

"How convinced are you that global warming is happening?"

\begin{tabular}{|l|c|}
\hline & Percent \\
\hline Convinced (net) & 81 \\
\hline Completely & 43 \\
\hline Mostly & 38 \\
\hline Not convinced (net) & 19 \\
\hline Not very & 14 \\
\hline Not at all & 5 \\
\hline Don't know & 0 \\
\hline Total & 100 \\
\hline
\end{tabular}

\section{Table 2: Cause of Global Warming}

"If global warming is happening, do you think it is due more to the normal cycles in the Earth's environment, or more to human activity such as burning fossil fuels?"

\begin{tabular}{|l|c|}
\hline & Percent \\
\hline Human activity & 55 \\
\hline Normal cycles & 37 \\
\hline Don't know & 7 \\
\hline Not happening & 1 \\
\hline Total & 100 \\
\hline
\end{tabular}




\section{Table 3: Current Impacts}

"Some people say that global warming is already impacting Alaska. Do you strongly agree, somewhat agree, somewhat disagree, or strongly disagree that global warming is already causing or making the following things worse?"

\begin{tabular}{|l|c|c|c|c|c|c|c|c|}
\hline & $\begin{array}{c}\text { Agree } \\
\text { (net) }\end{array}$ & $\begin{array}{c}\text { Strongly } \\
\text { agree }\end{array}$ & $\begin{array}{c}\text { Somewhat } \\
\text { agree }\end{array}$ & $\begin{array}{c}\text { Disagree } \\
\text { (net) }\end{array}$ & $\begin{array}{c}\text { Somewhat } \\
\text { disagree }\end{array}$ & $\begin{array}{c}\text { Strongly } \\
\text { disagree }\end{array}$ & $\begin{array}{c}\text { Don't } \\
\text { know }\end{array}$ & Total \\
\hline $\begin{array}{l}\text { Loss of sea ice in } \\
\text { the Arctic Ocean }\end{array}$ & 83 & 53 & 30 & 13 & 8 & 6 & 4 & 100 \\
\hline $\begin{array}{l}\text { Melting } \\
\text { permafrost }\end{array}$ & 82 & 53 & 29 & 14 & 7 & 6 & 5 & 100 \\
\hline $\begin{array}{l}\text { Coastlines } \\
\text { eroding and } \\
\text { flooding }\end{array}$ & 74 & 41 & 34 & 20 & 11 & 9 & 5 & 100 \\
\hline Forest fires & 72 & 41 & 31 & 25 & 15 & 10 & 3 & 100 \\
\hline $\begin{array}{l}\text { More } \\
\text { unpredictable } \\
\text { weather }\end{array}$ & 70 & 37 & 33 & 28 & 17 & 11 & 2 & 100 \\
\hline $\begin{array}{l}\text { Changing bird } \\
\text { migrations }\end{array}$ & 64 & 30 & 34 & 24 & 14 & 11 & 12 & 100 \\
\hline $\begin{array}{l}\text { Ice storms and } \\
\text { freezing rain }\end{array}$ & 56 & 21 & 35 & 36 & 20 & 16 & 8 & 100 \\
\hline
\end{tabular}




\section{LOCAL CHANGES IN CLIMATE}

\section{Table 4: Local Temperatures}

"Over the past five years, would you say that average temperatures in your local area have gotten warmer, colder, or stayed about the same?"

\begin{tabular}{|l|c|}
\hline & Percent \\
\hline Warmer & 67 \\
\hline About the same & 21 \\
\hline Colder & 5 \\
\hline Don't know & 7 \\
\hline Total & 100 \\
\hline
\end{tabular}

Table 5: Cause of Local Temperature Changes

[IF WARMER OR COLDER IN PREVIOUS QUESTION] "In your opinion, how much of the change in your local temperatures is due to global warming?"

\begin{tabular}{|l|c|}
\hline & Percent \\
\hline All of it & 14 \\
\hline Most of it & 40 \\
\hline Some of it & 39 \\
\hline None & 4 \\
\hline Don't know & 3 \\
\hline Total & 100 \\
\hline
\end{tabular}




\section{PUBLIC RISK PERCEPTIONS}

\section{Table 6: Good or Bad for Alaska}

"Overall, do you think that global warming will be very good, somewhat good, somewhat bad or very bad for Alaska?"

\begin{tabular}{|l|c|}
\hline & Percent \\
\hline Bad (net) & 67 \\
\hline Very bad & 38 \\
\hline Somewhat bad & 29 \\
\hline Good (net) & 26 \\
\hline Somewhat good & 23 \\
\hline Very good & 3 \\
\hline Don't know & 7 \\
\hline Total & 100 \\
\hline
\end{tabular}

\section{Table 7: Seriousness}

"How serious of a threat is global warming to:"

\begin{tabular}{|l|c|c|c|c|c|c|c|c|}
\hline & $\begin{array}{c}\text { Serious } \\
\text { (net) }\end{array}$ & $\begin{array}{c}\text { Very } \\
\text { serious }\end{array}$ & $\begin{array}{c}\text { Somewhat } \\
\text { serious }\end{array}$ & $\begin{array}{c}\text { Not } \\
\text { serious } \\
\text { (net) }\end{array}$ & $\begin{array}{c}\text { Not very } \\
\text { serious }\end{array}$ & $\begin{array}{c}\text { Not at all } \\
\text { serious }\end{array}$ & $\begin{array}{c}\text { Don't } \\
\text { know }\end{array}$ & Total \\
\hline $\begin{array}{l}\text { Plants and } \\
\text { animals }\end{array}$ & 76 & 47 & 29 & 22 & 13 & 9 & 2 & 100 \\
\hline People in Alaska & 71 & 32 & 39 & 27 & 16 & 12 & 2 & 100 \\
\hline $\begin{array}{l}\text { People in the } \\
\text { United States }\end{array}$ & 71 & 29 & 42 & 25 & 14 & 11 & 4 & 100 \\
\hline $\begin{array}{l}\text { People in other } \\
\text { countries }\end{array}$ & 68 & 31 & 36 & 24 & 13 & 11 & 8 & 100 \\
\hline $\begin{array}{l}\text { Your local } \\
\text { community }\end{array}$ & 59 & 20 & 40 & 39 & 23 & 16 & 2 & 100 \\
\hline $\begin{array}{l}\text { You and your } \\
\text { family }\end{array}$ & 55 & 18 & 37 & 44 & 28 & 16 & 1 & 100 \\
\hline
\end{tabular}




\section{Table 8: Timing}

"When do you think global warming will start to have dangerous impacts on people in Alaska?"

\begin{tabular}{|l|c|}
\hline & Percent \\
\hline It is dangerous now & 24 \\
\hline In 10 years & 19 \\
\hline In 25 years & 18 \\
\hline In 50 years & 15 \\
\hline In 100 years & 8 \\
\hline Never & 10 \\
\hline Don't know & 6 \\
\hline Total & 100 \\
\hline
\end{tabular}

Table 9: Likelihood of Impacts on Alaska

"In Alaska, how likely do you think it is that each of the following will occur, during the next 50 years due to global warming?"

\begin{tabular}{|l|c|c|c|c|c|c|c|c|}
\hline & $\begin{array}{c}\text { Likely } \\
\text { (net) }\end{array}$ & $\begin{array}{c}\text { Very } \\
\text { likely }\end{array}$ & $\begin{array}{c}\text { Somewhat } \\
\text { likely }\end{array}$ & $\begin{array}{c}\text { Unlikely } \\
\text { (net) }\end{array}$ & $\begin{array}{c}\text { Somewhat } \\
\text { unlikely }\end{array}$ & $\begin{array}{c}\text { Very } \\
\text { unlikely }\end{array}$ & $\begin{array}{c}\text { Don't } \\
\text { know }\end{array}$ & Total \\
\hline $\begin{array}{l}\text { Permanent loss of } \\
\text { summer sea ice in the } \\
\text { Arctic Ocean }\end{array}$ & 78 & 51 & 26 & 19 & 9 & 10 & 4 & 100 \\
\hline $\begin{array}{l}\text { More comfortable } \\
\text { temperatures }\end{array}$ & 73 & 27 & 46 & 23 & 13 & 10 & 4 & 100 \\
\hline $\begin{array}{l}\text { Flooding of towns and } \\
\text { villages }\end{array}$ & 73 & 39 & 35 & 25 & 11 & 14 & 2 & 100 \\
\hline Worse storms & 68 & 33 & 35 & 30 & 17 & 12 & 3 & 100 \\
\hline Fewer salmon & 67 & 37 & 30 & 26 & 14 & 13 & 7 & 100 \\
\hline $\begin{array}{l}\text { Extinction of the polar } \\
\text { bear }\end{array}$ & 65 & 36 & 29 & 29 & 15 & 14 & 6 & 100 \\
\hline $\begin{array}{l}\text { Increased rates of } \\
\text { disease }\end{array}$ & 61 & 22 & 39 & 36 & 18 & 18 & 4 & 100 \\
\hline More tourism & 60 & 30 & 30 & 35 & 19 & 16 & 5 & 100 \\
\hline Food shortages & 56 & 20 & 36 & 42 & 22 & 21 & 2 & 100 \\
\hline
\end{tabular}




\section{POLICY PREFERENCES AND BEHAVIOR}

\section{Table 10: Urgency of Action}

"Do you think global warming is an urgent problem that requires immediate government action, or a longer-term problem that requires more study before government action is taken?"

\begin{tabular}{|l|c|}
\hline & Percent \\
\hline Urgent problem & 48 \\
\hline Longer-term problem & 45 \\
\hline Not a problem & 4 \\
\hline Don't know & 3 \\
\hline Total & 100 \\
\hline
\end{tabular}

\section{Table 11: Native Villages and Coastal Erosion}

"Four native villages are in imminent danger from coastal erosion and flooding. Do you think the State of Alaska should help to:"

\begin{tabular}{|l|c|}
\hline & Percent \\
\hline Move these villages to safer ground & 41 \\
\hline Maintain these villages in their current locations & 21 \\
\hline Let the villages take care of themselves & 17 \\
\hline Relocate the people to other parts of Alaska & 15 \\
\hline Don't know & 5 \\
\hline Total & 100 \\
\hline
\end{tabular}




\section{Table 12: Mitigation}

"For each of the following, please tell me whether you strongly favor, somewhat favor, somewhat oppose, or strongly oppose it as a way for the federal government to try to reduce future global warming."

\begin{tabular}{|l|c|c|c|c|c|c|c|c|}
\hline & $\begin{array}{c}\text { Favor } \\
\text { (net) }\end{array}$ & $\begin{array}{c}\text { Strongly } \\
\text { favor }\end{array}$ & $\begin{array}{c}\text { Somewhat } \\
\text { favor }\end{array}$ & $\begin{array}{c}\text { Oppose } \\
\text { (net) }\end{array}$ & $\begin{array}{c}\text { Somewhat } \\
\text { oppose }\end{array}$ & $\begin{array}{c}\text { Strongly } \\
\text { oppose }\end{array}$ & $\begin{array}{c}\text { Don't } \\
\text { know }\end{array}$ & Total \\
\hline $\begin{array}{l}\text { Regulate } \\
\text { greenhouse gas } \\
\text { emissions from } \\
\text { power plants }\end{array}$ & 81 & 53 & 28 & 15 & 9 & 6 & 4 & 100 \\
\hline $\begin{array}{l}\text { Sign international } \\
\text { treaties to reduce } \\
\text { greenhouse gas } \\
\text { emissions }\end{array}$ & 71 & 45 & 26 & 23 & 12 & 11 & 6 & 100 \\
\hline $\begin{array}{l}\text { Increase taxes on } \\
\text { gasoline so people } \\
\text { either drive less or } \\
\text { buy cars that use } \\
\text { less gas }\end{array}$ & 30 & 14 & 16 & 69 & 11 & 58 & 1 & 100 \\
\hline $\begin{array}{l}\text { Increase taxes on } \\
\text { electricity so people } \\
\text { use less of it }\end{array}$ & 28 & 10 & 17 & 71 & 16 & 55 & 2 & 100 \\
\hline
\end{tabular}




\section{Table 13: Trust}

"Do you trust or distrust the following individuals and groups to tell you the truth about global warming -- strongly trust, somewhat trust, somewhat distrust, strongly distrust?"

\begin{tabular}{|l|c|c|c|c|c|c|c|c|}
\hline & $\begin{array}{c}\text { Trust } \\
\text { (net) }\end{array}$ & $\begin{array}{c}\text { Strongly } \\
\text { trust }\end{array}$ & $\begin{array}{c}\text { Somewhat } \\
\text { trust }\end{array}$ & $\begin{array}{c}\text { Distrust } \\
\text { (net) }\end{array}$ & $\begin{array}{c}\text { Somewhat } \\
\text { distrust }\end{array}$ & $\begin{array}{c}\text { Strongly } \\
\text { distrust }\end{array}$ & $\begin{array}{c}\text { Don't } \\
\text { know }\end{array}$ & Total \\
\hline Family and friends & 86 & 25 & 62 & 12 & 10 & 2 & 2 & 100 \\
\hline Scientists & 82 & 41 & 41 & 16 & 10 & 6 & 2 & 100 \\
\hline $\begin{array}{l}\text { Environmental } \\
\text { organizations }\end{array}$ & 63 & 18 & 45 & 37 & 15 & 22 & 1 & 100 \\
\hline The news media & 48 & 4 & 44 & 52 & 24 & 28 & 1 & 100 \\
\hline $\begin{array}{l}\text { Religious } \\
\text { organizations }\end{array}$ & 40 & 6 & 34 & 53 & 23 & 30 & 7 & 100 \\
\hline $\begin{array}{l}\text { President George W. } \\
\text { Bush }\end{array}$ & 33 & 6 & 27 & 66 & 17 & 49 & 2 & 100 \\
\hline Alaskan politicians & 29 & 3 & 25 & 70 & 25 & 45 & 2 & 100 \\
\hline Corporations & 23 & 3 & 21 & 75 & 30 & 45 & 2 & 100 \\
\hline
\end{tabular}




\section{Table 14: Individual Behavior}

"How likely are you to do the following because of your concerns about global warming?"

- Buy energy-efficient appliances (refrigerators, stoves, dishwashers, etc.)

- Make your views on global warming clear to politicians (by writing, telephoning, sending e-mails, signing petitions, etc.)

- Join, donate money to, or volunteer with an organization working on issues related to global warming.

\begin{tabular}{|l|c|c|c|c|c|c|c|c|}
\hline & $\begin{array}{c}\text { Likely } \\
\text { (net) }\end{array}$ & $\begin{array}{c}\text { Very } \\
\text { likely }\end{array}$ & $\begin{array}{c}\text { Somewhat } \\
\text { likely }\end{array}$ & $\begin{array}{c}\text { Unlikely } \\
\text { (net) }\end{array}$ & $\begin{array}{c}\text { Somewhat } \\
\text { unlikely }\end{array}$ & $\begin{array}{c}\text { Very } \\
\text { unlikely }\end{array}$ & $\begin{array}{c}\text { Don't } \\
\text { know }\end{array}$ & Total \\
\hline $\begin{array}{l}\text { Buy energy-efficient } \\
\text { appliances }\end{array}$ & 73 & 45 & 28 & 26 & 13 & 13 & 1 & 100 \\
\hline $\begin{array}{l}\text { Make your views } \\
\text { clear to politicians }\end{array}$ & 60 & 24 & 35 & 40 & 15 & 25 & 1 & 100 \\
\hline $\begin{array}{l}\text { Join, donate money, } \\
\text { or volunteer }\end{array}$ & 48 & 16 & 32 & 51 & 19 & 32 & 1 & 100 \\
\hline
\end{tabular}

\section{Table 15: Talk about Global Warming}

"How often do you talk about global warming with your friends and family?"

\begin{tabular}{|l|c|}
\hline & Percent \\
\hline Very often & 18 \\
\hline Occasionally & 44 \\
\hline Rarely & 30 \\
\hline Never & 8 \\
\hline Total & 100 \\
\hline
\end{tabular}




\section{Selected Results by Political Party, Political Ideology, and Region ${ }^{6}$}

\section{PUBLIC BELIEFS ABOUT GLOBAL WARMING}

\section{Table 16: Is it Happening?}

"How convinced are you that global warming is happening?"

\begin{tabular}{|l|l|c|c|c|c|c|}
\hline \multirow{2}{*}{} & \multicolumn{4}{|c|}{ Political Party } & \multirow{2}{*}{ Total } \\
\cline { 2 - 6 } & Republicans & No Party & Democrats & Other & \\
\hline \multirow{3}{*}{ Respondent is: } & Completely convinced & $28.2 \%$ & $40.9 \%$ & $56.4 \%$ & $55.3 \%$ & $43.3 \%$ \\
\cline { 2 - 7 } & Mostly convinced & $36.9 \%$ & $43.3 \%$ & $34.9 \%$ & $28.2 \%$ & $37.7 \%$ \\
\cline { 2 - 7 } & Not so convinced & $26.1 \%$ & $10.6 \%$ & $7.7 \%$ & $12.9 \%$ & $14.3 \%$ \\
\cline { 2 - 7 } & Not at all convinced & $8.4 \%$ & $5.2 \%$ & $.6 \%$ & $3.5 \%$ & $4.5 \%$ \\
\cline { 2 - 7 } & Don't Know & $.3 \%$ & & $.3 \%$ & $.2 \%$ & $100.0 \%$ \\
\hline Total & $100.0 \%$ & $100.0 \%$ & $100.0 \%$ & $100.0 \%$ & 100.0 \\
\hline
\end{tabular}

\begin{tabular}{|l|l|c|c|c|c|}
\hline \multirow{2}{*}{} & \multicolumn{2}{c|}{ Political Ideology } & \multirow{2}{*}{ Total } \\
\cline { 3 - 6 } & Conservative & Moderate & \multicolumn{2}{c|}{ Liberal } & \\
\hline \multirow{3}{*}{ Respondent is: } & Completely convinced & $27.2 \%$ & $42.6 \%$ & $58.7 \%$ & $43.5 \%$ \\
\cline { 2 - 5 } & Mostly convinced & $40.8 \%$ & $41.3 \%$ & $31.0 \%$ & $37.8 \%$ \\
\cline { 2 - 6 } & Not so convinced & $22.3 \%$ & $13.5 \%$ & $7.4 \%$ & $14.0 \%$ \\
\cline { 2 - 6 } & Not at all convinced & $9.8 \%$ & $2.0 \%$ & $2.9 \%$ & $4.4 \%$ \\
\cline { 2 - 6 } & Don't Know & & $.5 \%$ & & $.2 \%$ \\
\hline Total & $100.0 \%$ & $100.0 \%$ & $100.0 \%$ & $100.0 \%$ \\
\hline
\end{tabular}

\begin{tabular}{|c|c|c|c|c|c|c|}
\hline & & \multicolumn{4}{|c|}{ Region } & \multirow{2}{*}{ Total } \\
\hline & & Urban & Northern Urban & Semi-urban & Rural & \\
\hline \multirow{5}{*}{ Respondent is: } & Completely convinced & $40.5 \%$ & $51.0 \%$ & $47.2 \%$ & $39.0 \%$ & $43.3 \%$ \\
\hline & Mostly convinced & $36.8 \%$ & $32.0 \%$ & $40.7 \%$ & $45.0 \%$ & $37.7 \%$ \\
\hline & Not so convinced & $16.5 \%$ & $12.4 \%$ & $10.3 \%$ & $13.0 \%$ & $14.2 \%$ \\
\hline & Not at all convinced & $6.0 \%$ & $4.6 \%$ & $1.4 \%$ & $3.0 \%$ & $4.5 \%$ \\
\hline & Don't Know & $.2 \%$ & & $.5 \%$ & & $.2 \%$ \\
\hline \multicolumn{2}{|l|}{ Total } & $100.0 \%$ & $100.0 \%$ & $100.0 \%$ & $100.0 \%$ & $100.0 \%$ \\
\hline
\end{tabular}

\footnotetext{
${ }^{6}$ See the "Definitions" section at the end of the report for descriptions of these categories.
} 


\section{Table 17: Cause of Global Warming}

"If global warming is happening, do you think it is due more to the normal cycles in the Earth's environment, or more to human activity such as burning fossil fuels?"

\begin{tabular}{|l|l|l|l|l|l|c|}
\hline \multicolumn{2}{|c|}{} & \multicolumn{3}{|c|}{ Political Party } & \multirow{2}{*}{ Total } \\
\cline { 3 - 7 } & More to normal cycles & $55.6 \%$ & $37.7 \%$ & $20.4 \%$ & $27.1 \%$ & $36.5 \%$ \\
\hline \multirow{3}{*}{ Global warming is due: } & More to human activity & $37.8 \%$ & $56.2 \%$ & $71.2 \%$ & $51.8 \%$ & $55.3 \%$ \\
\cline { 2 - 7 } & Is not happening & $.7 \%$ & $1.2 \%$ & $2.6 \%$ & & $1.4 \%$ \\
\cline { 2 - 7 } & Don't know & $5.9 \%$ & $4.9 \%$ & $5.8 \%$ & $21.2 \%$ & $6.8 \%$ \\
\hline Total & $100.0 \%$ & $100.0 \%$ & $100.0 \%$ & $100.0 \%$ & $100.0 \%$ \\
\hline
\end{tabular}

\begin{tabular}{|l|l|c|c|c|c|}
\hline \multirow{2}{*}{} & \multicolumn{3}{|c|}{ Political ldeology } & \multirow{2}{*}{ Total } \\
\cline { 3 - 6 } & More to normal cycles & $56.0 \%$ & $34.5 \%$ & $23.5 \%$ & $36.9 \%$ \\
\hline \multirow{3}{*}{ Global warming is due: } & More to human activity & $41.0 \%$ & $55.0 \%$ & $68.5 \%$ & $55.5 \%$ \\
\cline { 2 - 6 } & Is not happening & $.8 \%$ & $.8 \%$ & $2.3 \%$ & $1.2 \%$ \\
\cline { 2 - 6 } & Don't know & $2.3 \%$ & $9.7 \%$ & $5.8 \%$ & $6.4 \%$ \\
\hline Total & & $100.0 \%$ & $100.0 \%$ & $100.0 \%$ & $100.0 \%$ \\
\hline
\end{tabular}

\begin{tabular}{|l|l|l|c|c|c|c|}
\hline \multicolumn{2}{|c|}{} & \multicolumn{4}{c|}{ Region } & \multirow{2}{*}{ Total } \\
\cline { 3 - 7 } & More to normal cycles & $38.0 \%$ & $47.1 \%$ & $29.4 \%$ & $27.6 \%$ & $36.5 \%$ \\
\hline \multirow{3}{*}{ Global warming is due: } & More to human activity & $53.5 \%$ & $45.1 \%$ & $62.6 \%$ & $66.3 \%$ & $55.4 \%$ \\
\cline { 2 - 7 } & Is not happening & & $1.3 \%$ & $3.7 \%$ & $4.1 \%$ & $1.4 \%$ \\
\cline { 2 - 7 } & Don't know & $8.5 \%$ & $6.5 \%$ & $4.2 \%$ & $2.0 \%$ & $6.7 \%$ \\
\hline Total & $100.0 \%$ & $100.0 \%$ & $100.0 \%$ & $100.0 \%$ & $100.0 \%$ \\
\hline
\end{tabular}




\section{LOCAL CHANGES IN CLIMATE}

\section{Table 18: Local Temperatures}

"Over the past five years, would you say that average temperatures in your local area have gotten warmer, colder, or stayed about the same?"

\begin{tabular}{|l|l|c|c|c|c|c|}
\hline \multicolumn{2}{|c|}{} & \multicolumn{3}{|c|}{ Political Party } & \multirow{2}{*}{ Total } \\
\cline { 2 - 6 } & Are warmer & Republicans & No Party & Democrats & Other & \\
\hline \multirow{3}{*}{ Average temperatures: } & Colder & $58.2 \%$ & $74.8 \%$ & $75.1 \%$ & $83.3 \%$ & $71.2 \%$ \\
\cline { 2 - 7 } & Stayed about the same & $5.5 \%$ & $6.0 \%$ & $4.6 \%$ & $2.6 \%$ & $5.1 \%$ \\
\cline { 2 - 7 } & Don't Know & $.4 \%$ & $18.9 \%$ & $17.4 \%$ & $14.1 \%$ & $22.6 \%$ \\
\hline Total & $100.0 \%$ & $100.0 \%$ & $100.0 \%$ & $100.0 \%$ & $100.0 \%$ \\
\hline
\end{tabular}

\begin{tabular}{|l|l|c|c|c|c|}
\hline \multirow{2}{*}{} & \multicolumn{2}{|c|}{ Political Ideology } & \multirow{2}{*}{ Total } \\
\cline { 2 - 6 } & Are warmer & Conservative & Moderate & Liberal & \\
\hline \multirow{3}{*}{ Average temperatures: } & Colder & $58.7 \%$ & $72.8 \%$ & $80.6 \%$ & $71.6 \%$ \\
\cline { 2 - 6 } & Stayed about the same & $5.5 \%$ & $4.7 \%$ & $5.2 \%$ & $5.1 \%$ \\
\cline { 2 - 6 } & Don't Know & $35.7 \%$ & $20.2 \%$ & $13.9 \%$ & $22.2 \%$ \\
\hline Total & & $2.3 \%$ & $.3 \%$ & $1.1 \%$ \\
\hline
\end{tabular}

\begin{tabular}{|l|l|c|c|c|c|c|}
\hline \multicolumn{2}{|c|}{} & \multicolumn{4}{c|}{ Region } & \multirow{2}{*}{ Total } \\
\cline { 2 - 7 } & Are warmer & Urban & Northern Urban & Semi-urban & Rural & \\
\hline \multirow{3}{*}{ Average temperatures: } & Colder & $74.1 \%$ & $73.3 \%$ & $65.2 \%$ & $65.6 \%$ & $71.3 \%$ \\
\cline { 2 - 7 } & Stayed about the same & $3.4 \%$ & $3.7 \%$ & $6.8 \%$ & $11.8 \%$ & $5.0 \%$ \\
\cline { 2 - 7 } & Don't Know & $.2 \%$ & $23.0 \%$ & $23.2 \%$ & $22.6 \%$ & $22.6 \%$ \\
\hline Total & $100.0 \%$ & $100.0 \%$ & $100.0 \%$ & $100.0 \%$ & $100.0 \%$ \\
\hline
\end{tabular}


Table 19: Cause of Local Temperature Changes

[IF WARMER OR COLDER IN PREVIOUS QUESTION]

"In your opinion, how much of the change in your local temperatures is due to global warming?"

\begin{tabular}{|l|l|c|c|c|c|c|}
\hline \multirow{2}{*}{} & \multicolumn{3}{|c|}{ Political Party } & \multirow{2}{*}{ Total } \\
\cline { 3 - 6 } & Republicans & No Party & Democrats & Other & \\
\hline \multirow{3}{*}{$\begin{array}{l}\text { Change due to } \\
\text { global warning: }\end{array}$} & All it & $9.4 \%$ & $14.6 \%$ & $16.3 \%$ & $14.1 \%$ & $13.9 \%$ \\
\cline { 2 - 6 } & Most of it & $38.9 \%$ & $36.7 \%$ & $47.6 \%$ & $23.9 \%$ & $39.5 \%$ \\
\cline { 2 - 6 } & Some of it & $38.3 \%$ & $38.6 \%$ & $32.5 \%$ & $56.3 \%$ & $38.2 \%$ \\
\cline { 2 - 6 } & None & $8.3 \%$ & $7.1 \%$ & $.8 \%$ & $2.8 \%$ & $5.0 \%$ \\
\cline { 2 - 6 } & Don't Know/No Answer & $5.0 \%$ & $3.0 \%$ & $2.8 \%$ & $2.8 \%$ & $3.4 \%$ \\
\hline \multirow{2}{*}{ Total } & $100.0 \%$ & $100.0 \%$ & $100.0 \%$ & $100.0 \%$ & $100.0 \%$ \\
\hline
\end{tabular}

\begin{tabular}{|l|l|c|c|c|c|}
\hline \multirow{2}{*}{} & \multicolumn{3}{c|}{ Political Ideology } & \multirow{2}{*}{ Total } \\
\cline { 2 - 5 } & All of it & Conservative & Moderate & Liberal & \\
\hline \multirow{3}{*}{$\begin{array}{l}\text { Change due to } \\
\text { global warning: }\end{array}$} & Most of it & $14.8 \%$ & $8.6 \%$ & $19.2 \%$ & $13.8 \%$ \\
\cline { 2 - 5 } & Some of it & $30.2 \%$ & $40.2 \%$ & $46.2 \%$ & $40.0 \%$ \\
\cline { 2 - 5 } & None & $43.2 \%$ & $41.5 \%$ & $31.5 \%$ & $38.4 \%$ \\
\cline { 2 - 6 } & Don't Know/No Answer & $7.1 \%$ & $7.0 \%$ & $.4 \%$ & $4.7 \%$ \\
\hline \multirow{2}{*}{ Total } & $100.0 \%$ & $100.0 \%$ & $100.0 \%$ & $100.0 \%$ \\
\hline
\end{tabular}

\begin{tabular}{|l|l|l|c|c|c|c|}
\hline \multirow{2}{*}{} & \multicolumn{4}{c|}{ Region } & \multirow{2}{*}{ Total } \\
\cline { 3 - 6 } & All of it & $12.7 \%$ & $18.2 \%$ & $9.9 \%$ & $20.8 \%$ & $13.8 \%$ \\
\hline \multirow{3}{*}{$\begin{array}{l}\text { Change due to } \\
\text { global warning: }\end{array}$} & Most of it & $36.6 \%$ & $36.4 \%$ & $46.7 \%$ & $46.8 \%$ & $39.6 \%$ \\
\cline { 2 - 6 } & Some of it & $41.1 \%$ & $32.2 \%$ & $39.5 \%$ & $29.9 \%$ & $38.3 \%$ \\
\cline { 2 - 6 } & None & $6.9 \%$ & $5.8 \%$ & $1.3 \%$ & $4.9 \%$ \\
\cline { 2 - 7 } & Don't Know/No Answer & $2.6 \%$ & $7.4 \%$ & $2.6 \%$ & $2.6 \%$ & $3.4 \%$ \\
\hline Total & $100.0 \%$ & $100.0 \%$ & $100.0 \%$ & $100.0 \%$ & $100.0 \%$ \\
\hline
\end{tabular}




\section{PUBLIC RISK PERCEPTIONS}

\section{Table 20: Good or Bad for Alaska}

"Overall, do you think that global warming will be very good, somewhat good, somewhat bad or very bad for Alaska?"

\begin{tabular}{|l|l|c|c|c|c|c|}
\hline \multirow{2}{*}{} & \multicolumn{3}{|c|}{ Political Party } & \multirow{2}{*}{ Total } \\
\cline { 3 - 7 } & Republicans & No Party & Democrats & Other & \\
\hline \multirow{4}{*}{$\begin{array}{l}\text { Overall, global } \\
\text { warming will be: }\end{array}$} & Very good & $3.5 \%$ & $4.6 \%$ & $1.9 \%$ & $2.4 \%$ & $3.3 \%$ \\
\cline { 2 - 7 } & Somewhat good & $29.6 \%$ & $21.3 \%$ & $18.2 \%$ & $22.4 \%$ & $22.8 \%$ \\
\cline { 2 - 7 } & Somewhat bad & $35.5 \%$ & $26.4 \%$ & $25.2 \%$ & $34.1 \%$ & $29.3 \%$ \\
\cline { 2 - 7 } & Very bad & $22.6 \%$ & $42.2 \%$ & $49.5 \%$ & $27.1 \%$ & $37.7 \%$ \\
\cline { 2 - 7 } & Don't Know/No Answer & $8.7 \%$ & $5.5 \%$ & $5.1 \%$ & $14.1 \%$ & $7.0 \%$ \\
\hline \multirow{2}{*}{ Total } & $100.0 \%$ & $100.0 \%$ & $100.0 \%$ & $100.0 \%$ & $100.0 \%$ \\
\hline
\end{tabular}

\begin{tabular}{|l|l|c|c|c|c|}
\hline \multirow{2}{*}{} & \multicolumn{3}{c|}{ Political Ideology } & \multirow{2}{*}{ Total } \\
\cline { 2 - 6 } & Conservative & Moderate & Liberal & \\
\hline \multirow{3}{*}{$\begin{array}{l}\text { Overall, global } \\
\text { warming will be: }\end{array}$} & Very good & $4.5 \%$ & $2.1 \%$ & $3.2 \%$ & $3.1 \%$ \\
\cline { 2 - 5 } & Somewhat good & $25.7 \%$ & $23.1 \%$ & $19.6 \%$ & $22.7 \%$ \\
\cline { 2 - 6 } & Somewhat bad & $37.4 \%$ & $29.0 \%$ & $23.5 \%$ & $29.5 \%$ \\
\cline { 2 - 6 } & Very bad & $21.9 \%$ & $39.2 \%$ & $50.5 \%$ & $38.1 \%$ \\
\cline { 2 - 6 } & Don't Know/No Answer & $10.6 \%$ & $6.7 \%$ & $3.2 \%$ & $6.6 \%$ \\
\hline \multirow{2}{*}{ Total } & $100.0 \%$ & $100.0 \%$ & $100.0 \%$ & $100.0 \%$ \\
\hline
\end{tabular}

\begin{tabular}{|l|l|c|c|c|c|c|}
\hline \multicolumn{2}{|c|}{} & \multicolumn{4}{c|}{ Region } & \multirow{2}{*}{ Total } \\
\cline { 3 - 7 } \multicolumn{2}{|c|}{} & Urban & Northern Urban & Semi-urban & Rural & \\
\hline \multirow{3}{*}{$\begin{array}{l}\text { Overall, global } \\
\text { warming will be: }\end{array}$} & Very good & $.7 \%$ & $6.6 \%$ & $2.3 \%$ & $15.2 \%$ & $3.3 \%$ \\
\cline { 2 - 7 } & Somewhat good & $21.4 \%$ & $28.3 \%$ & $20.4 \%$ & $26.3 \%$ & $22.7 \%$ \\
\cline { 2 - 7 } & Somewhat bad & $34.2 \%$ & $25.7 \%$ & $20.8 \%$ & $25.3 \%$ & $29.2 \%$ \\
\cline { 2 - 7 } & Very bad & $35.5 \%$ & $31.6 \%$ & $50.0 \%$ & $32.3 \%$ & $37.7 \%$ \\
\cline { 2 - 7 } & Don't Know/No Answer & $8.2 \%$ & $7.9 \%$ & $6.5 \%$ & $1.0 \%$ & $7.1 \%$ \\
\hline \multirow{2}{*}{ Total } & $100.0 \%$ & $100.0 \%$ & $100.0 \%$ & $100.0 \%$ & $100.0 \%$ \\
\hline
\end{tabular}




\section{Table 21: Seriousness - You and Your Family}

"How serious of a threat is global warming to you and your family?"

\begin{tabular}{|l|l|c|c|c|c|c|}
\hline \multicolumn{2}{|c|}{} & \multicolumn{4}{|c|}{ Political Party } & \multirow{2}{*}{ Total } \\
\cline { 3 - 7 } & Republicans & No Party & Democrats & Other & \\
\hline \multirow{5}{*}{ Family } & Very serious & $10.8 \%$ & $19.8 \%$ & $22.8 \%$ & $17.9 \%$ & $18.0 \%$ \\
\cline { 2 - 7 } & Somewhat serious & $26.8 \%$ & $37.7 \%$ & $45.8 \%$ & $34.5 \%$ & $36.9 \%$ \\
\cline { 2 - 7 } & Not very serious & $39.0 \%$ & $24.3 \%$ & $25.0 \%$ & $15.5 \%$ & $28.0 \%$ \\
\cline { 2 - 7 } & Not at all serious & $23.0 \%$ & $17.3 \%$ & $4.2 \%$ & $29.8 \%$ & $15.9 \%$ \\
\cline { 2 - 7 } & Don't Know/No Answer & $.3 \%$ & $.9 \%$ & $2.2 \%$ & $2.4 \%$ & $1.3 \%$ \\
\hline \multirow{3}{*}{ Total } & & $100.0 \%$ & $100.0 \%$ & $100.0 \%$ & $100.0 \%$ & $100.0 \%$ \\
\hline
\end{tabular}

\begin{tabular}{|l|l|c|c|c|c|}
\hline \multicolumn{2}{|c|}{} & \multicolumn{2}{|c|}{ Political Ideology } & \multirow{2}{*}{ Total } \\
\cline { 3 - 5 } & Conservative & Moderate & Liberal & \\
\hline \multirow{4}{*}{ Family } & Very serious & $9.1 \%$ & $19.7 \%$ & $24.5 \%$ & $18.3 \%$ \\
\cline { 2 - 5 } & Somewhat serious & $24.2 \%$ & $38.2 \%$ & $46.5 \%$ & $37.0 \%$ \\
\cline { 2 - 5 } & Not very serious & $40.8 \%$ & $25.1 \%$ & $21.0 \%$ & $28.1 \%$ \\
\cline { 2 - 5 } & Not at all serious & $25.3 \%$ & $14.6 \%$ & $7.7 \%$ & $15.3 \%$ \\
\cline { 2 - 6 } & Don't Know/No Answer & $.8 \%$ & $2.3 \%$ & $.3 \%$ & $1.2 \%$ \\
\hline \multirow{3}{*}{ Total } & & $100.0 \%$ & $100.0 \%$ & $100.0 \%$ & $100.0 \%$ \\
\hline
\end{tabular}

\begin{tabular}{|l|l|c|c|c|c|c|}
\hline \multicolumn{2}{|c|}{} & \multicolumn{4}{c|}{ Region } & \multirow{2}{*}{ Total } \\
\cline { 3 - 7 } & Urban & Northern Urban & Semi-urban & Rural & \\
\hline \multirow{3}{*}{ Family } & Very serious & $13.2 \%$ & $15.7 \%$ & $26.8 \%$ & $29.6 \%$ & $18.0 \%$ \\
\cline { 2 - 7 } & Somewhat serious & $34.5 \%$ & $36.6 \%$ & $40.8 \%$ & $41.8 \%$ & $36.9 \%$ \\
\cline { 2 - 7 } & Not very serious & $32.0 \%$ & $29.4 \%$ & $21.1 \%$ & $17.3 \%$ & $27.9 \%$ \\
\cline { 2 - 7 } & Not at all serious & $19.3 \%$ & $17.6 \%$ & $8.0 \%$ & $11.2 \%$ & $15.9 \%$ \\
\cline { 2 - 7 } & Don't Know/No Answer & $.9 \%$ & $.7 \%$ & $3.3 \%$ & $1.3 \%$ \\
\hline \multirow{3}{*}{ Total } & $100.0 \%$ & $100.0 \%$ & $100.0 \%$ & $100.0 \%$ & $100.0 \%$ \\
\hline
\end{tabular}




\section{Table 22: Seriousness - Your Local Community}

"How serious of a threat is global warming to your local community?"

\begin{tabular}{|l|l|c|c|c|c|c|}
\hline \multicolumn{2}{|c|}{} & \multicolumn{3}{|c|}{ Political Party } & \multirow{2}{*}{ Total } \\
\cline { 2 - 6 } & Republicans & No Party & Democrats & Other & \\
\hline \multirow{3}{*}{ Local community } & Very serious & $10.1 \%$ & $22.0 \%$ & $28.5 \%$ & $11.8 \%$ & $19.8 \%$ \\
\cline { 2 - 7 } & Somewhat serious & $35.5 \%$ & $42.4 \%$ & $41.0 \%$ & $36.5 \%$ & $39.5 \%$ \\
\cline { 2 - 7 } & Not very serious & $26.1 \%$ & $21.6 \%$ & $24.4 \%$ & $16.5 \%$ & $23.3 \%$ \\
\cline { 2 - 7 } & Not at all serious & $27.2 \%$ & $11.9 \%$ & $5.1 \%$ & $29.4 \%$ & $15.6 \%$ \\
\cline { 2 - 7 } & Don't Know/No Answer & $1.0 \%$ & $2.1 \%$ & $1.0 \%$ & $5.9 \%$ & $1.8 \%$ \\
\hline \multirow{2}{*}{ Total } & $100.0 \%$ & $100.0 \%$ & $100.0 \%$ & $100.0 \%$ & $100.0 \%$ \\
\hline
\end{tabular}

\begin{tabular}{|l|l|c|c|c|c|}
\hline \multirow{2}{*}{} & \multicolumn{3}{c|}{ Political Ideology } & \multirow{2}{*}{ Total } \\
\cline { 2 - 5 } & Very serious & Conservative & Moderate & Liberal & \\
\hline \multirow{3}{*}{ Local community } & Somewhat serious & $31.2 \%$ & $44.2 \%$ & $39.5 \%$ & $39.2 \%$ \\
\cline { 2 - 5 } & Not very serious & $27.1 \%$ & $23.5 \%$ & $20.6 \%$ & $23.6 \%$ \\
\cline { 2 - 5 } & Not at all serious & $30.1 \%$ & $10.0 \%$ & $9.0 \%$ & $15.2 \%$ \\
\cline { 2 - 6 } & Don't Know/No Answer & $1.1 \%$ & $2.6 \%$ & $1.3 \%$ & $1.8 \%$ \\
\hline \multirow{2}{*}{ Total } & $100.0 \%$ & $100.0 \%$ & $100.0 \%$ & $100.0 \%$ \\
\hline
\end{tabular}

\begin{tabular}{|l|l|c|c|c|c|c|}
\hline \multicolumn{2}{|c|}{} & \multicolumn{3}{c|}{ Region } & \multirow{2}{*}{ Total } \\
\cline { 3 - 6 } \multicolumn{1}{|c|}{} & Urban & Northern Urban & Semi-urban & Rural & \\
\hline \multirow{3}{*}{ Local community } & Very serious & $16.3 \%$ & $16.2 \%$ & $27.9 \%$ & $28.3 \%$ & $19.9 \%$ \\
\cline { 2 - 7 } & Somewhat serious & $39.1 \%$ & $40.9 \%$ & $38.6 \%$ & $40.4 \%$ & $39.4 \%$ \\
\cline { 2 - 6 } & Not very serious & $24.5 \%$ & $20.1 \%$ & $24.7 \%$ & $19.2 \%$ & $23.3 \%$ \\
\cline { 2 - 7 } & Not at all serious & $18.8 \%$ & $20.1 \%$ & $7.0 \%$ & $9.1 \%$ & $15.6 \%$ \\
\cline { 2 - 7 } & Don't Know/No Answer & $1.3 \%$ & $2.6 \%$ & $1.9 \%$ & $3.0 \%$ & $1.8 \%$ \\
\hline Total & $100.0 \%$ & $100.0 \%$ & $100.0 \%$ & $100.0 \%$ & $100.0 \%$ \\
\hline
\end{tabular}




\section{Table 23: Seriousness - People in Alaska}

"How serious of a threat is global warming to people in Alaska?"

\begin{tabular}{|l|l|c|c|c|c|c|}
\hline \multicolumn{2}{|c|}{} & \multicolumn{3}{|c|}{ Political Party } & \multirow{2}{*}{ Total } \\
\cline { 3 - 6 } & Republicans & No Party & Democrats & Other & \\
\hline \multirow{3}{*}{ People in Alaska } & Very serious & $20.3 \%$ & $31.3 \%$ & $45.7 \%$ & $26.2 \%$ & $32.2 \%$ \\
\cline { 2 - 7 } & Somewhat serious & $41.6 \%$ & $41.0 \%$ & $35.1 \%$ & $32.1 \%$ & $38.6 \%$ \\
\cline { 2 - 7 } & Not very serious & $18.9 \%$ & $15.8 \%$ & $13.4 \%$ & $13.1 \%$ & $15.7 \%$ \\
\cline { 2 - 7 } & Not at all serious & $18.5 \%$ & $9.7 \%$ & $2.6 \%$ & $27.4 \%$ & $11.5 \%$ \\
\cline { 2 - 7 } & Don't Know/No Answer & $.7 \%$ & $2.1 \%$ & $3.2 \%$ & $1.2 \%$ & $2.0 \%$ \\
\hline Total & $100.0 \%$ & $100.0 \%$ & $100.0 \%$ & $100.0 \%$ & $100.0 \%$ \\
\hline
\end{tabular}

\begin{tabular}{|l|l|c|c|c|c|}
\hline \multicolumn{2}{|c|}{} & \multicolumn{3}{c|}{ Political Ideology } & \multirow{2}{*}{ Total } \\
\cline { 2 - 6 } & Conservative & Moderate & Liberal & \\
\hline \multirow{3}{*}{ People in Alaska } & Very serious & $18.8 \%$ & $30.2 \%$ & $47.4 \%$ & $32.6 \%$ \\
\cline { 2 - 6 } & Somewhat serious & $41.0 \%$ & $42.5 \%$ & $31.3 \%$ & $38.5 \%$ \\
\cline { 2 - 6 } & Not very serious & $17.7 \%$ & $15.6 \%$ & $14.8 \%$ & $15.9 \%$ \\
\cline { 2 - 6 } & Not at all serious & $21.1 \%$ & $9.0 \%$ & $5.5 \%$ & $11.2 \%$ \\
\cline { 2 - 6 } & Don't Know/No Answer & $1.5 \%$ & $2.8 \%$ & $1.0 \%$ & $1.9 \%$ \\
\hline Total & $100.0 \%$ & $100.0 \%$ & $100.0 \%$ & $100.0 \%$ \\
\hline
\end{tabular}

\begin{tabular}{|l|l|c|c|c|c|c|}
\hline \multicolumn{2}{|c|}{} & \multicolumn{4}{c|}{ Region } & \multirow{2}{*}{ Total } \\
\cline { 3 - 7 } \multicolumn{1}{|c|}{} & Urban & Northern Urban & Semi-urban & \multirow{2}{*}{ Rural } & \\
\hline \multirow{3}{*}{ People in Alaska } & Very serious & $31.8 \%$ & $27.6 \%$ & $35.0 \%$ & $35.4 \%$ & $32.2 \%$ \\
\cline { 2 - 7 } & Somewhat serious & $40.5 \%$ & $35.5 \%$ & $36.0 \%$ & $39.4 \%$ & $38.7 \%$ \\
\cline { 2 - 7 } & Not very serious & $13.4 \%$ & $21.7 \%$ & $17.3 \%$ & $15.2 \%$ & $15.6 \%$ \\
\cline { 2 - 7 } & Not at all serious & $13.7 \%$ & $13.8 \%$ & $5.6 \%$ & $9.1 \%$ & $11.6 \%$ \\
\cline { 2 - 7 } & Don't Know/No Answer & $.5 \%$ & $1.3 \%$ & $6.1 \%$ & $1.0 \%$ & $1.9 \%$ \\
\hline Total & $100.0 \%$ & $100.0 \%$ & $100.0 \%$ & $100.0 \%$ & $100.0 \%$ \\
\hline
\end{tabular}




\section{Table 24: Timing}

"When do you think global warming will start to have dangerous impacts on people in Alaska?"

\begin{tabular}{|l|l|c|c|c|c|c|}
\hline \multicolumn{2}{|c|}{} & \multicolumn{4}{|c|}{ Political Party } & \multirow{2}{*}{ Total } \\
\cline { 3 - 7 } \multicolumn{1}{|c|}{} & Republicans & No Party & Democrats & Other & \\
\hline \multirow{4}{*}{ It is dangerous now } & $16.4 \%$ & $26.7 \%$ & $31.6 \%$ & $15.3 \%$ & $24.4 \%$ \\
\cline { 2 - 7 } & In 10 years & $16.7 \%$ & $18.8 \%$ & $19.2 \%$ & $24.7 \%$ & $18.8 \%$ \\
\cline { 2 - 7 } & In 25 years & $13.6 \%$ & $21.0 \%$ & $21.7 \%$ & $8.2 \%$ & $18.0 \%$ \\
\cline { 2 - 7 } & In 50 years & $12.5 \%$ & $13.1 \%$ & $15.3 \%$ & $29.4 \%$ & $15.0 \%$ \\
\cline { 2 - 7 } & In 100 years & $12.9 \%$ & $4.3 \%$ & $6.4 \%$ & $17.6 \%$ & $8.5 \%$ \\
\cline { 2 - 7 } & Never & $18.5 \%$ & $11.2 \%$ & $1.6 \%$ & $2.4 \%$ & $9.6 \%$ \\
\cline { 2 - 7 } & Don't Know/No Answer & $9.4 \%$ & $4.9 \%$ & $4.2 \%$ & $2.4 \%$ & $5.7 \%$ \\
\hline Total & & $100.0 \%$ & $100.0 \%$ & $100.0 \%$ & $100.0 \%$ & $100.0 \%$ \\
\hline
\end{tabular}

\begin{tabular}{|l|l|c|c|c|c|}
\hline \multicolumn{2}{|c|}{} & \multicolumn{3}{c|}{ Political Ideology } & \multirow{2}{*}{ Total } \\
\cline { 3 - 5 } & Conservative & Moderate & Liberal & \\
\hline \multirow{5}{*}{ It is dangerous now } & $16.6 \%$ & $21.8 \%$ & $35.6 \%$ & $24.8 \%$ \\
\cline { 2 - 5 } & In $\mathbf{1 0}$ years & $12.1 \%$ & $22.8 \%$ & $20.1 \%$ & $19.0 \%$ \\
\cline { 2 - 5 } & In $\mathbf{2 5}$ years & $16.2 \%$ & $18.5 \%$ & $18.4 \%$ & $17.8 \%$ \\
\cline { 2 - 5 } & In $\mathbf{5 0}$ years & $12.8 \%$ & $19.0 \%$ & $12.3 \%$ & $15.1 \%$ \\
\cline { 2 - 5 } & In 100 years & $14.7 \%$ & $5.6 \%$ & $6.5 \%$ & $8.4 \%$ \\
\cline { 2 - 5 } & Never & $20.0 \%$ & $7.2 \%$ & $3.2 \%$ & $9.4 \%$ \\
\cline { 2 - 5 } & Don't Know/No Answer & $7.5 \%$ & $5.1 \%$ & $3.9 \%$ & $5.4 \%$ \\
\hline \multirow{3}{*}{ Total } & $100.0 \%$ & $100.0 \%$ & $100.0 \%$ & $100.0 \%$ \\
\hline
\end{tabular}

\begin{tabular}{|l|l|c|c|c|c|c|}
\hline \multicolumn{2}{|c|}{} & \multicolumn{4}{|c|}{ Region } & \multirow{2}{*}{ Total } \\
\cline { 3 - 7 } \multicolumn{1}{|c|}{} & Urban & Northern Urban & Semi-urban & Rural & \\
\hline \multirow{4}{*}{ Alaskans } & It is dangerous now & $20.3 \%$ & $17.8 \%$ & $37.4 \%$ & $30.6 \%$ & $24.5 \%$ \\
\cline { 2 - 7 } & In 10 years & $18.1 \%$ & $21.1 \%$ & $15.9 \%$ & $26.5 \%$ & $18.9 \%$ \\
\cline { 2 - 7 } & In 25 years & $16.5 \%$ & $19.1 \%$ & $17.3 \%$ & $26.5 \%$ & $18.0 \%$ \\
\cline { 2 - 7 } & In 50 years & $15.2 \%$ & $12.5 \%$ & $18.2 \%$ & $10.2 \%$ & $14.9 \%$ \\
\cline { 2 - 7 } & In 100 years & $10.7 \%$ & $11.2 \%$ & $2.8 \%$ & $4.1 \%$ & $8.5 \%$ \\
\cline { 2 - 7 } & Never & $12.8 \%$ & $11.2 \%$ & $3.7 \%$ & $1.0 \%$ & $9.5 \%$ \\
\cline { 2 - 7 } & Don't Know/No Answer & $6.5 \%$ & $7.2 \%$ & $4.7 \%$ & $1.0 \%$ & $5.7 \%$ \\
\hline \multirow{2}{*}{ Total } & $100.0 \%$ & $100.0 \%$ & $100.0 \%$ & $100.0 \%$ & $100.0 \%$ \\
\hline
\end{tabular}




\section{POLICY PREFERENCES AND BEHAVIOR}

Table 25: Urgency of Action

"Do you think global warming is an urgent problem that requires immediate government action, or a longer-term problem that requires more study before government action is taken?"

\begin{tabular}{|l|l|c|c|c|c|c|}
\hline \multicolumn{2}{|c|}{} & \multicolumn{3}{|c|}{ Political Party } & \multirow{2}{*}{ Total } \\
\cline { 2 - 7 } & Republicans & No Party & Democrats & \multirow{2}{*}{ Other } & \\
\hline \multirow{3}{*}{$\begin{array}{l}\text { Global warming } \\
\text { requires: }\end{array}$} & $\begin{array}{l}\text { Immediate government } \\
\text { action }\end{array}$ & $28.9 \%$ & $51.4 \%$ & $62.2 \%$ & $44.0 \%$ & $47.7 \%$ \\
\cline { 2 - 7 } & Longer-term problem & $64.1 \%$ & $41.6 \%$ & $32.4 \%$ & $44.0 \%$ & $45.4 \%$ \\
\cline { 2 - 7 } & Not a problem & $5.6 \%$ & $4.6 \%$ & $1.9 \%$ & $1.2 \%$ & $3.8 \%$ \\
\cline { 2 - 7 } & Don't Know/No Answer & $1.4 \%$ & $2.4 \%$ & $3.5 \%$ & $10.7 \%$ & $3.2 \%$ \\
\hline Total & $100.0 \%$ & $100.0 \%$ & $100.0 \%$ & $100.0 \%$ & $100.0 \%$ \\
\hline
\end{tabular}

\begin{tabular}{|l|l|c|c|c|c|}
\hline \multicolumn{2}{|c|}{} & \multicolumn{2}{c|}{ Political Ideology } & \multirow{2}{*}{ Total } \\
\cline { 3 - 5 } & $\begin{array}{l}\text { Immediate government } \\
\text { action }\end{array}$ & $28.7 \%$ & $50.0 \%$ & $63.3 \%$ & $48.4 \%$ \\
\cline { 2 - 5 } & Longer-term problem & $61.1 \%$ & $44.1 \%$ & $32.8 \%$ & $45.1 \%$ \\
\cline { 2 - 5 } \multirow{3}{*}{$\begin{array}{l}\text { Global warming } \\
\text { requires: }\end{array}$} & Not a problem & $8.7 \%$ & $1.0 \%$ & $2.6 \%$ & $3.6 \%$ \\
\cline { 2 - 5 } & Don't Know/No Answer & $1.5 \%$ & $4.9 \%$ & $1.3 \%$ & $2.8 \%$ \\
\hline \multirow{2}{*}{ Total } & $100.0 \%$ & $100.0 \%$ & $100.0 \%$ & $100.0 \%$ \\
\hline
\end{tabular}

\begin{tabular}{|l|l|c|c|c|c|c|}
\hline \multirow{2}{*}{} & \multicolumn{4}{c|}{ Region } & \multirow{2}{*}{ Total } \\
\cline { 3 - 6 } & $\begin{array}{l}\text { Immediate government } \\
\text { action }\end{array}$ & $45.5 \%$ & $38.6 \%$ & $58.4 \%$ & $51.5 \%$ & $47.7 \%$ \\
\cline { 2 - 6 } & Longer-term problem & $48.2 \%$ & $51.0 \%$ & $35.0 \%$ & $41.4 \%$ & $45.2 \%$ \\
\cline { 2 - 6 } & Not a problem & $3.3 \%$ & $7.8 \%$ & $1.9 \%$ & $5.1 \%$ & $3.8 \%$ \\
\cline { 2 - 7 } $\begin{array}{l}\text { Global warming } \\
\text { requires: }\end{array}$ & Don't Know/No Answer & $3.1 \%$ & $2.6 \%$ & $4.7 \%$ & $2.0 \%$ & $3.2 \%$ \\
\hline Total & $100.0 \%$ & $100.0 \%$ & $100.0 \%$ & $100.0 \%$ & $100.0 \%$ \\
\hline
\end{tabular}




\section{Table 26: Native Villages and Coastal Erosion}

"Four native villages are in imminent danger from coastal erosion and flooding. Do you think the State of Alaska should help to:"

\begin{tabular}{|l|l|c|c|c|c|c|}
\hline \multicolumn{2}{|c|}{} & \multicolumn{3}{|c|}{ Political Party } & \multirow{2}{*}{ Total } \\
\cline { 3 - 7 } \multicolumn{1}{|c|}{} & Republicans & No Party & Democrats & Other & \\
\hline \multirow{3}{*}{ The State should: } & Maintain villages & $19.2 \%$ & $28.0 \%$ & $17.5 \%$ & $10.7 \%$ & $20.8 \%$ \\
\cline { 2 - 7 } & Move villages & $44.3 \%$ & $34.8 \%$ & $46.5 \%$ & $35.7 \%$ & $41.2 \%$ \\
\cline { 2 - 7 } & Relocate people & $12.5 \%$ & $13.7 \%$ & $20.7 \%$ & $9.5 \%$ & $15.2 \%$ \\
\cline { 2 - 7 } & Let villages alone & $21.6 \%$ & $19.8 \%$ & $8.9 \%$ & $25.0 \%$ & $17.4 \%$ \\
\cline { 2 - 7 } & Don't Know/No Answer & $2.4 \%$ & $3.7 \%$ & $6.4 \%$ & $19.0 \%$ & $5.4 \%$ \\
\hline \multirow{2}{*}{ Total } & $100.0 \%$ & $100.0 \%$ & $100.0 \%$ & $100.0 \%$ & $100.0 \%$ \\
\hline
\end{tabular}

\begin{tabular}{|l|l|c|c|c|c|}
\hline \multicolumn{2}{|c|}{} & \multicolumn{3}{c|}{ Political Ideology } & \multirow{2}{*}{ Total } \\
\cline { 2 - 5 } & Maintain villages & Conservative & Moderate & Liberal & \\
\hline \multirow{3}{*}{ The State should: } & Move villages & $21.4 \%$ & $20.2 \%$ & $21.5 \%$ & $21.0 \%$ \\
\cline { 2 - 5 } & Relocate people & $13.2 \%$ & $13.6 \%$ & $17.7 \%$ & $14.8 \%$ \\
\cline { 2 - 6 } & Let villages alone & $25.2 \%$ & $14.3 \%$ & $14.5 \%$ & $17.4 \%$ \\
\cline { 2 - 6 } & Don't Know/No Answer & $3.8 \%$ & $8.4 \%$ & $3.5 \%$ & $5.6 \%$ \\
\hline \multirow{3}{*}{ Total } & $100.0 \%$ & $100.0 \%$ & $100.0 \%$ & $100.0 \%$ \\
\hline
\end{tabular}

\begin{tabular}{|c|c|c|c|c|c|c|}
\hline & & \multicolumn{4}{|c|}{ Region } & \multirow{2}{*}{ Total } \\
\hline & & Urban & Northern Urban & Semi-urban & Rural & \\
\hline \multirow{5}{*}{ The State should: } & Maintain villages & $13.4 \%$ & $13.7 \%$ & $33.5 \%$ & $46.5 \%$ & $20.9 \%$ \\
\hline & Move villages & $45.7 \%$ & $47.1 \%$ & $30.2 \%$ & $31.3 \%$ & $41.3 \%$ \\
\hline & Relocate people & $14.3 \%$ & $18.3 \%$ & $16.3 \%$ & $12.1 \%$ & $15.1 \%$ \\
\hline & Let villages alone & $19.4 \%$ & $11.1 \%$ & $19.5 \%$ & $10.1 \%$ & $17.3 \%$ \\
\hline & Don't Know/No Answer & $7.1 \%$ & $9.8 \%$ & $.5 \%$ & & $5.4 \%$ \\
\hline \multicolumn{2}{|l|}{ Total } & $100.0 \%$ & $100.0 \%$ & $100.0 \%$ & $100.0 \%$ & $100.0 \%$ \\
\hline
\end{tabular}




\section{Table 27: Mitigation - International Treaties}

"For each of the following, please tell me whether you strongly favor, somewhat favor, somewhat oppose, or strongly oppose it as a way for the federal government to try to reduce future global warming:"

"Sign international treaties to reduce greenhouse gas emissions"

\begin{tabular}{|c|c|c|c|c|c|c|}
\hline & & \multicolumn{4}{|c|}{ Political Party } & \multirow{2}{*}{ Total } \\
\hline & & Republicans & No Party & Democrats & Other & \\
\hline \multirow{5}{*}{$\begin{array}{l}\text { Sign international } \\
\text { treaties to reduce } \\
\text { greenhouse gas }\end{array}$} & Strongly favor & $34.5 \%$ & $39.9 \%$ & $57.2 \%$ & $50.6 \%$ & $44.6 \%$ \\
\hline & Somewhat favor & $26.5 \%$ & $27.4 \%$ & $28.1 \%$ & $14.1 \%$ & $26.3 \%$ \\
\hline & Somewhat oppose & $16.7 \%$ & $11.9 \%$ & $4.5 \%$ & $23.5 \%$ & $11.9 \%$ \\
\hline & Strongly oppose & $19.5 \%$ & $9.8 \%$ & $4.2 \%$ & $11.8 \%$ & $11.0 \%$ \\
\hline & Don't Know/No Answer & $2.8 \%$ & $11.0 \%$ & $6.1 \%$ & & $6.2 \%$ \\
\hline \multicolumn{2}{|l|}{ Total } & $100.0 \%$ & $100.0 \%$ & $100.0 \%$ & $100.0 \%$ & $100.0 \%$ \\
\hline
\end{tabular}

\begin{tabular}{|l|l|c|c|c|c|}
\hline \multirow{2}{*}{} & \multicolumn{3}{c|}{ Political Ideology } & \multirow{2}{*}{ Total } \\
\cline { 2 - 5 } & Strongly favor & Conservative & Moderate & Liberal & \\
\hline \multirow{3}{*}{$\begin{array}{l}\text { Sign international } \\
\text { treaties to reduce } \\
\text { greenhouse gas }\end{array}$} & Somewhat favor & $19.5 \%$ & $43.2 \%$ & $57.1 \%$ & $45.0 \%$ \\
\cline { 2 - 6 } & Somewhat oppose & $17.3 \%$ & $11.0 \%$ & $9.0 \%$ & $12.1 \%$ \\
\cline { 2 - 6 } & Strongly oppose & $24.8 \%$ & $4.9 \%$ & $6.8 \%$ & $11.0 \%$ \\
\cline { 2 - 6 } & Don't Know/No Answer & $4.9 \%$ & $7.4 \%$ & $3.9 \%$ & $5.6 \%$ \\
\hline \multirow{2}{*}{ Total } & $100.0 \%$ & $100.0 \%$ & $100.0 \%$ & $100.0 \%$ \\
\hline
\end{tabular}

\begin{tabular}{|l|l|l|c|c|c|c|}
\hline \multirow{2}{*}{} & \multicolumn{4}{c|}{ Region } & \multirow{2}{*}{ Total } \\
\cline { 3 - 7 } & Urban & Northern Urban & Semi-urban & Rural & \\
\hline \multirow{3}{*}{$\begin{array}{l}\text { Sign international } \\
\text { treaties to reduce } \\
\text { greenhouse gas }\end{array}$} & Strongly favor & $48.6 \%$ & $41.8 \%$ & $44.9 \%$ & $27.3 \%$ & $44.7 \%$ \\
\cline { 2 - 7 } & Somewhat favor & $18.1 \%$ & $29.4 \%$ & $30.8 \%$ & $56.6 \%$ & $26.2 \%$ \\
\cline { 2 - 7 } & Somewhat oppose & $15.8 \%$ & $11.1 \%$ & $5.6 \%$ & $6.1 \%$ & $12.0 \%$ \\
\cline { 2 - 7 } & Strongly oppose & $12.7 \%$ & $13.7 \%$ & $6.1 \%$ & $6.1 \%$ & $10.8 \%$ \\
\cline { 2 - 7 } & Don't Know/No Answer & $4.9 \%$ & $3.9 \%$ & $12.6 \%$ & $4.0 \%$ & $6.3 \%$ \\
\hline Total & $100.0 \%$ & $100.0 \%$ & $100.0 \%$ & $100.0 \%$ & $100.0 \%$ \\
\hline
\end{tabular}


Table 28: Mitigation - Regulation of Power Plants

"Regulate greenhouse gas emissions from power plants."

\begin{tabular}{|l|l|c|c|c|c|c|}
\hline \multirow{2}{*}{} & \multicolumn{3}{|c|}{ Political Party } & \multirow{2}{*}{ Total } \\
\cline { 3 - 7 } & Republicans & No Party & Democrats & Other & \\
\hline \multirow{3}{*}{$\begin{array}{l}\text { Regulate greenhouse } \\
\text { gas emissions from } \\
\text { power plants }\end{array}$} & Strongly favor & $41.1 \%$ & $50.6 \%$ & $66.5 \%$ & $54.7 \%$ & $53.1 \%$ \\
\cline { 2 - 7 } & Somewhat favor & $40.4 \%$ & $26.4 \%$ & $22.4 \%$ & $11.6 \%$ & $27.9 \%$ \\
\cline { 2 - 7 } & Somewhat oppose & $11.1 \%$ & $9.4 \%$ & $4.2 \%$ & $20.9 \%$ & $9.3 \%$ \\
\cline { 2 - 7 } & Strongly oppose & $6.3 \%$ & $7.9 \%$ & $3.5 \%$ & $9.3 \%$ & $6.2 \%$ \\
\cline { 2 - 7 } & Don't Know/No Answer & $1.0 \%$ & $5.8 \%$ & $3.5 \%$ & $3.5 \%$ & $3.5 \%$ \\
\hline Total & $100.0 \%$ & $100.0 \%$ & $100.0 \%$ & $100.0 \%$ & $100.0 \%$ \\
\hline
\end{tabular}

\begin{tabular}{|l|l|c|c|c|c|}
\hline \multirow{2}{*}{} & \multicolumn{3}{c|}{ Political Ideology } & \multirow{2}{*}{ Total } \\
\cline { 3 - 6 } & Strongly favor & $41.0 \%$ & $52.8 \%$ & $66.8 \%$ & $54.0 \%$ \\
\hline \multirow{3}{*}{$\begin{array}{l}\text { Regulate greenhouse } \\
\text { gas emissions from } \\
\text { power plants }\end{array}$} & Somewhat favor & $35.0 \%$ & $29.2 \%$ & $19.7 \%$ & $27.7 \%$ \\
\cline { 2 - 5 } & Somewhat oppose & $12.8 \%$ & $10.5 \%$ & $4.5 \%$ & $9.2 \%$ \\
\cline { 2 - 5 } & Strongly oppose & $9.4 \%$ & $4.4 \%$ & $5.8 \%$ & $6.2 \%$ \\
\cline { 2 - 5 } & Don't Know/No Answer & $1.9 \%$ & $3.1 \%$ & $3.2 \%$ & $2.8 \%$ \\
\hline \multirow{2}{*}{ Total } & & $100.0 \%$ & $100.0 \%$ & $100.0 \%$ & $100.0 \%$ \\
\hline
\end{tabular}

\begin{tabular}{|l|l|l|c|c|c|c|}
\hline \multicolumn{2}{|c|}{} & \multicolumn{4}{c|}{ Region } & \multirow{2}{*}{ Total } \\
\cline { 3 - 7 } & Urban & Northern Urban & Semi-urban & \multirow{2}{*}{ Rural } & \\
\hline \multirow{3}{*}{$\begin{array}{l}\text { Regulate greenhouse } \\
\text { gas emissions from } \\
\text { power plants }\end{array}$} & Strongly favor & $60.5 \%$ & $49.7 \%$ & $46.3 \%$ & $33.3 \%$ & $53.2 \%$ \\
\cline { 2 - 7 } & Somewhat favor & $20.3 \%$ & $32.0 \%$ & $33.6 \%$ & $50.5 \%$ & $27.8 \%$ \\
\cline { 2 - 7 } & Somewhat oppose & $12.5 \%$ & $8.5 \%$ & $3.7 \%$ & $4.0 \%$ & $9.2 \%$ \\
\cline { 2 - 7 } & Strongly oppose & $5.3 \%$ & $8.5 \%$ & $6.1 \%$ & $9.1 \%$ & $6.3 \%$ \\
\cline { 2 - 7 } & Don't Know/No Answer & $1.4 \%$ & $1.3 \%$ & $10.3 \%$ & $3.0 \%$ & $3.4 \%$ \\
\hline Total & $100.0 \%$ & $100.0 \%$ & $100.0 \%$ & $100.0 \%$ & $100.0 \%$ \\
\hline
\end{tabular}




\section{Table 29: Political Behavior}

"How likely are you to make your views on global warming clear to politicians (by writing, telephoning, sending e-mails, signing petitions, etc.)?"

\begin{tabular}{|c|c|c|c|c|c|c|}
\hline & \multicolumn{4}{|c|}{ Political Party } & \multirow{2}{*}{ Total } \\
\hline & & Republicans & No Party & Democrats & Other & \\
\hline \multirow{5}{*}{$\begin{array}{l}\text { Make your views } \\
\text { clear to politicians }\end{array}$} & Very likely & $18.2 \%$ & $19.6 \%$ & $35.5 \%$ & $21.2 \%$ & $24.2 \%$ \\
\hline & Somewhat likely & $34.3 \%$ & $35.5 \%$ & $36.4 \%$ & $32.9 \%$ & $35.2 \%$ \\
\hline & Somewhat unlikely & $19.6 \%$ & $17.4 \%$ & $9.3 \%$ & $8.2 \%$ & $14.7 \%$ \\
\hline & Very unlikely & $27.6 \%$ & $27.2 \%$ & $17.9 \%$ & $37.6 \%$ & $25.3 \%$ \\
\hline & Don't Know/No Answer & $.3 \%$ & $.3 \%$ & $1.0 \%$ & & $.5 \%$ \\
\hline \multicolumn{2}{|l|}{ Total } & $100.0 \%$ & $100.0 \%$ & $100.0 \%$ & $100.0 \%$ & $100.0 \%$ \\
\hline
\end{tabular}

\begin{tabular}{|l|l|c|c|c|c|}
\hline \multicolumn{2}{|c|}{} & \multicolumn{3}{c|}{ Political Ideology } & \multirow{2}{*}{ Total } \\
\cline { 2 - 6 } & Conservative & Moderate & Liberal & \\
\hline \multirow{4}{*}{$\begin{array}{l}\text { Make your views } \\
\text { clear to politicians }\end{array}$} & Very likely & $20.8 \%$ & $14.3 \%$ & $40.6 \%$ & $24.5 \%$ \\
\cline { 2 - 6 } & Somewhat likely & $34.3 \%$ & $40.4 \%$ & $29.4 \%$ & $35.2 \%$ \\
\cline { 2 - 6 } & Somewhat unlikely & $14.3 \%$ & $17.6 \%$ & $11.6 \%$ & $14.8 \%$ \\
\cline { 2 - 6 } & Very unlikely & $30.6 \%$ & $27.4 \%$ & $17.7 \%$ & $25.2 \%$ \\
\cline { 2 - 6 } & Don't Know/No Answer & & $.3 \%$ & $.6 \%$ & $.3 \%$ \\
\hline \multirow{2}{*}{ Total } & $100.0 \%$ & $100.0 \%$ & $100.0 \%$ & $100.0 \%$ \\
\hline
\end{tabular}

\begin{tabular}{|l|l|l|c|c|c|c|}
\hline \multirow{2}{*}{} & \multicolumn{4}{c|}{ Region } & \multirow{2}{*}{ Total } \\
\cline { 3 - 7 } & Urban & Northern Urban & Semi-urban & \multirow{2}{*}{ Rural } & \\
\hline \multirow{4}{*}{$\begin{array}{l}\text { Make your views } \\
\text { clear to politicians }\end{array}$} & Very likely & $27.9 \%$ & $31.4 \%$ & $14.5 \%$ & $15.0 \%$ & $24.3 \%$ \\
\cline { 2 - 7 } & Somewhat likely & $36.2 \%$ & $29.4 \%$ & $36.4 \%$ & $34.0 \%$ & $35.0 \%$ \\
\cline { 2 - 7 } & Somewhat unlikely & $14.5 \%$ & $19.6 \%$ & $13.1 \%$ & $12.0 \%$ & $14.7 \%$ \\
\cline { 2 - 7 } & Very unlikely & $21.0 \%$ & $19.6 \%$ & $35.0 \%$ & $36.0 \%$ & $25.2 \%$ \\
\cline { 2 - 7 } & Don't Know/No Answer & $.4 \%$ & & $.9 \%$ & $3.0 \%$ & $.7 \%$ \\
\hline Total & $100.0 \%$ & $100.0 \%$ & $100.0 \%$ & $100.0 \%$ & $100.0 \%$ \\
\hline
\end{tabular}




\section{Methodology}

These results come from a statewide survey entitled "Alaskan Opinions on Global Warming," which examined Alaskan risk perceptions, policy preferences, and behaviors regarding global climate change. From May 9 to June 29, 2006, 1,016 adults (18 and older) completed a 25 minute telephone survey, for a CASRO response rate of $74 \%$. If researchers had sought to interview every household in Alaska using the same questionnaire, the findings would differ from these overall survey results by no more than 3.1 percentage points in either direction. For results based on the total sample of respondents, one can say with $95 \%$ confidence that the maximum margin of sampling error is $+/-3.1 \%$.

Respondents from rural Alaska were oversampled to enable urban vs. rural and native vs. non-native analyses. The results represent a random sample of Alaska residents and are weighted ${ }^{7}$ to bring them in line with actual population proportions. This material is based upon work supported by the National Science Foundation and the Center for Environmental Decisions (CRED) at Columbia University under Grant No. SES 0345840. Any opinions, findings, and conclusions or recommendations expressed in this material are those of the authors and do not necessarily reflect the views of the National Science Foundation. The study was commissioned by Decision Research, and conducted by Craciun Research Group, Inc.

\section{Definitions}

\section{Political Party}

Republicans $=$ individuals who self-identify as Republican or leaning Republican Democrats $=$ individuals who self-identify as Democratic or leaning Democratic No Party $=$ individuals who do not identify with either Republicans or Democrats Other $=$ individuals who self-identify with other parties (e.g., Libertarian, Green, Independent Party)

\section{Political Ideology}

Conservatives $=$ self-identified as somewhat or very conservative Liberals $=$ self-identified as somewhat or very liberal Moderates $=$ self-identified as moderate, middle of the road

\footnotetext{
${ }^{7}$ Weighting causes slight anomalies such as the apparent increase in the total sample from 1016 to 1018.
} 


\section{Region}

Alaskans can, for the purpose of this study, be grouped into four regions based on the similarity of their lifestyles. Education, income levels, ethnicity, outdoor activities, politics, military service and frequency of attending church vary significantly in the four regions:

Urban, Anchorage and Mat-Su, includes more than half the population of

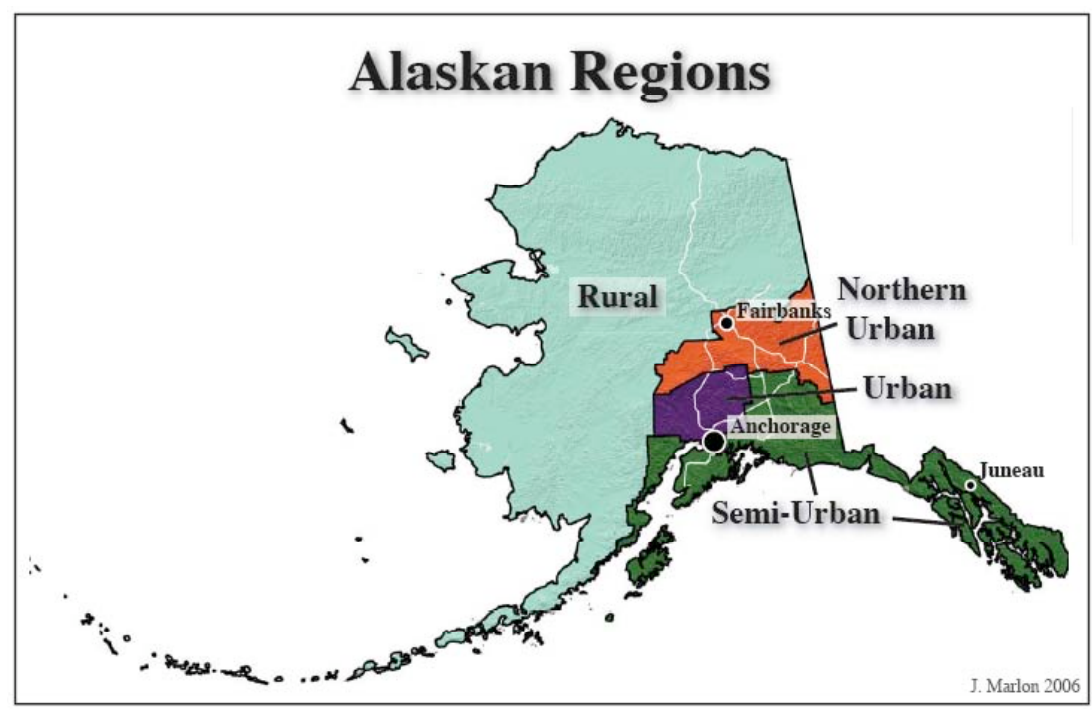
the state. Most of the households in this area contain people who work at jobs for wages, drive cars, and shop at supermarkets. In this sample, they have the highest median household incomes $(\$ 77,727)$ of the four areas, and the most college graduates (47\%, including $17 \%$ post-graduate degrees). Seventy-nine percent $(79 \%)$ of the population sampled in this area is Caucasian. Most of them $(76 \%)$ work indoors, and only $12 \%$ get half or more of their diet from wild foods gathered by the household. Except for gardening, they are the least likely of those sampled to engage in subsistence or sports activities outdoors. Thirty-two percent (32\%) consider themselves at least somewhat conservative politically, and $26 \%$ go to religious services weekly.

In the Northern Urban region, primarily Fairbanks and surrounding areas, the lifestyle is mostly, but not entirely, tied to roads and the modern mobile economy. They differ from Anchorage, Mat-Su in several respects. The population is much lower, the society more small-town. In the sample for this survey, the median income is lower than Anchorage, Mat-Su at $\$ 56,136$. The percentage of college graduates is slightly lower at $37 \%$ (including, however, $18 \%$ with post-graduate degrees). Seventy-eight percent $(78 \%)$ are Caucasian. Seventy-two percent $(72 \%)$ work indoors and $20 \%$ get half or more of their diet from wild foods. They differ from Anchorage, Mat-Su in the percentage who engage in outdoor activities; more of them consider fishing, hunting, and picking wild berries important to their lives. Thirty-nine percent $(39 \%)$ consider themselves conservative and $39 \%$ attend religious services weekly.

The Semi-Urban region includes the coastal area from Ketchikan in the Southeast to the Kenai Peninsula and Kodiak along the South central coast. Most of the people in these areas are connected by road or ferry and have roads within their locality. Many work for wages and/or do commercial fishing. Those sampled have a median income of $\$ 50,253$, and $15 \%$ have college degrees. Eighty-five percent $(85 \%)$ are Caucasian. Half $(49 \%)$ of them work outdoors, and $49 \%$ of the households get half or more of their diet from wild foods. Fishing, hunting and wild berry picking are important to more than 
six in ten of these households. Fourteen percent (14\%) are conservative politically, and $34 \%$ attend religious services weekly.

The mostly Rural region is distinguishable from all the others by the lack of roads. The vast area of Western and Northern Alaska is accessible from the population centers only by air. Comparatively few of these people work for wages; most households receive cash only from the State's Permanent Fund and/or dividends from their Native Corporations. These differences from the other areas are reflected in the median income of $\$ 28,060$, and in the lower percentage of college graduates $(10 \%)$. Twentyseven percent $(27 \%)$ are Caucasian, $68 \%$ Alaska Native. Fifty percent $(50 \%)$ work outdoors, and $83 \%$ of the households get half or more of their diet from subsistence. In this region, with few roads, $82 \%$ consider snow machining important to their lives. Thirteen percent $(13 \%)$ consider themselves politically conservative and $54 \%$ attend religious services weekly. 